\title{
Threshold uncertainty in discrete public good games: an experimental study
}

\author{
Michael McBride
}

Received: 15 April 2009 / Accepted: 13 October 2009 / Published online: 1 November 2009 (C) The Author(s) 2009. This article is published with open access at Springerlink.com

\begin{abstract}
A discrete public good is provided when total contributions equal or exceed the contribution threshold. Recent theoretical work shows that an increase in threshold uncertainty will increase (decrease) equilibrium contributions when the public good value is sufficiently high (low). In an experiment designed to test these predictions, I find only limited verification of the prediction. Using elicited beliefs data to represent subjects' beliefs, I find that behavior is not consistent with expected payoff maximization, however, contributions are increasing in subjects' subjective pivotalness. Thus, wider threshold uncertainty will sometimes-but not alwayshinder collective action.
\end{abstract}

Keywords Collective action $\cdot$ Participation $\cdot$ Experiments $\cdot$ Elicited beliefs

JEL Classification $\quad \mathrm{C} 72 \cdot \mathrm{C} 90 \cdot \mathrm{D} 80$

\begin{abstract}
Helpful comments were received from Stephen Morris, Ben Polak, David Pearce, Hongbin Cai, Andrew Schotter, Thomas Palfrey, Charles Plott, Dirk Bergemann, Leatt Yariv, Chris Udry, Pushkar Maitra, seminar participants at Yale University's game theory group, UCSB third-year seminar, BYU, UC Irvine, Ohio State, Stanford Graduate School of Business, participants at the 2004 Public Choice Society / Economic Science Association Meetings, and anonymous referees. Financial support was received from the Institution for Social and Policy Studies at Yale University, the California Social Science Experimental Laboratory (CASSEL) at UCLA, and the University of California, Irvine. Special thanks to the Social Science Experimental Laboratory at the California Institute of Technology and CASSEL for use of laboratory resources and to Yolanda Huang for programming assistance.
\end{abstract}

M. McBride $(\varangle)$

Department of Economics, University of California, Irvine,

3151 Social Science Plaza, Irvine, CA 92697-5100, USA

e-mail: mcbride@uci.edu 


\section{Introduction}

Many collective action scenarios, such as a multiple plaintiffs raising funds to achieve a commonly desired judicial ruling, neighborhood residents petitioning a local government to build a public project, and, more dramatically, plotters planning the size of their attempted coup, can be represented as discrete public good games. Specifically, a discrete public good is provided if contributions equal or exceed the required threshold level of contributions; otherwise, no good is provided. Since Olson's (1965) seminal work, researchers have examined how a number of factors, such as group size, excludability, selective incentives, punishment, and so on, inhibit or foster successful public good provision. One factor that potentially affects individuals' decisions to participate in a collective action is uncertainty ${ }^{1}$ about the threshold level of contributions needed for successful action. ${ }^{2}$ In the examples above, the plaintiffs might not know how much funds will be needed to fund a successful case, the neighborhood residents might not know how many signatures are needed to get the project built, and the coup plotters might not know how big their faction needs to be to overthrow the incumbent dictator.

Aware that threshold uncertainty affects an individual's strategic voluntary contribution decision, Nitzan and Romano (1990) and Suleiman (1997) extended the basic discrete public good model first studied by Palfrey and Rosenthal (1984) and Bagnoli and Lipman $(1989,1992)$ to include threshold uncertainty. They find that threshold uncertainty often results in inefficient equilibria because ex post excess contributions might be discarded or because contributions fall short of the threshold in equilibrium. ${ }^{3}$ More recently, however, McBride (2006) showed that the effect of an increase of uncertainty (as in a mean-preserving spread) on binary contribution decisions depends on the value of the public good. For example, in the neighborhood resident example mentioned earlier, suppose it is known exactly how many residents must petition the government to get the project approved and that the equilibrium outcome under perfect knowledge of the petition threshold is that enough petitions be made. McBride's result implies that if there is an increase in uncertainty about the petition threshold, then the number of petitions made to the government will actually increase when residents

\footnotetext{
1 With " risk" corresponding to known probabilities and " uncertainty" corresponding to unknown probabilities, the term risk is more appropriate here. However, I use the term uncertainty because the earlier work (e.g., Nitzan and Romano 1990) used that term.

2 Other types of uncertainty in public good games have also been considered. For example, Palfrey and Rosenthal (1988) consider uncertainty about others' degree of altruism; Palfrey and Rosenthal (1991) consider uncertainty about others' contribution costs; and Menezes et al. (2001) consider uncertainty about others' valuations of the public good. Morevoer, the discrete public good game with threshold uncertainty is similar to research on common pool resources with unknown pool size, e.g., Budescu et al. (1995).

3 Rebates and refunds may help mitigate some of the inefficiencies. For example, if money is refunded to contributors when the threshold is not met, then potential contributors do not risk paying for something and getting nothing in return, and if excess contributions above the threshold are rebated, then the risk of overpaying may disappear. However, free-rider problems may still exist because an individual still wants others to pay the costs instead of herself. Moreover, whether or not refunds and rebates resolve inefficiencies will depend on the way they are designed (e.g., Isaac et al. 1989; Marks and Croson 1998; Spencer et al. 2009). Also, rebates may not be technologically possible depending on the setting. Rebates seem a viable option if contributions are monetary, but less so if contributions are participatory in time and effort. When possible, though, they help mitigate some, even if not all, of the hindrances to successful collective action.
} 
highly value the project. On the other hand, if the project is not sufficiently valued, the increase in uncertainty will drive the number of petitions to zero.

An individual's marginal benefit of contributing a unit towards the public good depends on two things. The first is the value of the public good; as the public good value increases, so does the marginal benefit of contributing. The second is the probability that her contribution is pivotal in providing the good. If no refund will be given for overfunded or underfunded contributions, then an individual will only want to contribute if the probability that her contribution is pivotal in providing the good is sufficiently large. Intuitively, if an individual believes total contributions will not meet the threshold even with her contribution or if the total contributions already meet or exceed the threshold without her contribution, then she will not contribute. Key to McBride's result is that the probability of being pivotal is tied to the uncertainty about the threshold. As the uncertainty increases, the threshold is less likely to be some values but more likely to be others, and, the probability of being pivotal for certain contribution profiles changes accordingly. McBride shows that the probability of being pivotal increases (decreases) as uncertainty increases when the value of the public good is high (low), thereby driving up (down) contributions. The implication of this result is that threshold uncertainty need not inhibit successful provision of a discrete public good.

This paper presents results from an experiment designed to test this prediction. Previous experimental work has studied contributions in discrete public good games (also called step-level public goods, threshold public goods, or provision-point public goods), e.g., Offerman (1996), and Offerman et al. (1996) (see Ledyard 1995 for an earlier review of experimental work on public goods). Some of this work has examined threshold uncertainty. Wit and Wilke (1998) and Au (2004) conducted experiments with sequential contributions, and they find that contribution levels are lower under higher threshold uncertainty. Gustafsson, Biel, and Gärling (1998) report a similar finding in an analogous experiment with simultaneous contributions. Suleiman et al. (2001) find in a simultaneous contributions experiment that the effect of threshold uncertainty can depend on the mean of the threshold distribution. Unlike these earlier experiments, the experiment presented here varies the public good value, thereby allowing a test of the impact of changes in uncertainty at different public good values. The experiment also elicits subjects' beliefs about other subjects' contribution levels using a proper scoring rule. These data allow a closer examination of the subjects' decision making process because they can be used to infer a measure of the subjects' perceived pivotalness. Thus, unlike other experimental work, the experiment performed here can test McBride's (2006) prediction about the binary contribution decision.

Overall, the data provide some support, albeit weak, of the prediction. Contributions often increase as uncertainty increases when the public good value is high and decrease when the public good value is low. Yet, the prediction is not matched for every treatment. Thus, I consider two additional questions: why is the prediction verified to any degree, and why is that level of verification so weak? These questions can be addressed using the elicited beliefs data. An examination of these data reveal that subjects do update their reported beliefs in manners consistent with many learning models. This finding justifies using these data to proxy for subjects' true beliefs, which in turn allows me to calculate subjects' implied subjective probabilities 
of being pivotal. Conditioning on this subjective pivotalness, I show that subjects do not behave in a manner consistent in all ways with the model's implied decision rule. However, contribution behavior is consistent with one key feature of the decision rule: the likelihood a subject contributes is increasing in the subject's subjective pivotalness. In short, when making contribution decisions, individuals do act strategically in that they respond to pivotalness. This implies that even though the model's prediction about contribution levels is not strongly verified, the primary conclusion that threshold uncertainty need not inhibit collective action is supported.

\section{Model and predictions}

\subsection{Model set-up}

Consider a set of expected payoff maximizing players $N=\{1, \ldots, n\}, 2<n<\infty$. Players have identical strategy sets $S_{i}=\{0,1\}$. Choosing strategy $s_{i}=0$ is to be interpreted as not contributing, while choosing $s_{i}=1$ implies contributing. The cost of contributing one unit is $c>0$, the value of a provided public good is $v>0$, and both are the same for all individuals. The contribution threshold $t$ to provide the public good is chosen from a publicly known distribution cdf $F$ with pdf $f$ s.t. $F(0)=0$. Thus, the probability of providing the public $\operatorname{good}$ is $F\left(\sum_{j=1}^{n} s_{j}\right)$, and $i$ 's expected payoff given some profile $s$ of contribution choices is $u_{i}(s)=F\left(\sum_{j=1}^{n} s_{j}\right) v-s_{i} c .^{4}$ With $n, v, c$, and $F$ and all of the above commonly known, and assuming the players make their contribution choices simultaneously, we have a well-defined normal form game.

\subsection{Decision rule and equilibrium}

This game will generally have both pure and mixed equilibria. I review only the pure equilibria here, as the mixed equilibria will exhibit qualitatively similar features (McBride 2006).

An agent's decision in equilibrium will depend on her subjective probability of being pivotal in providing the public good. Denote $C_{-i}$ to be the set of contributing agents besides $i$, and also let it denote the number of contributing agents. The payoff matrix is

\begin{tabular}{c|c|c|c|} 
& \multicolumn{1}{c}{$\begin{array}{c}C_{-i}<t-1 \\
\text { (lost cause) }\end{array}$} & $\begin{array}{c}C_{-i}=t-1 \\
\text { (pivotal) }\end{array}$ & \multicolumn{1}{c}{$\begin{array}{c}C_{-i}>t-1 \\
\text { (redundant) }\end{array}$} \\
\cline { 2 - 4 }$s_{i}=1$ (contribute) & $-c$ & $v-c$ & $v-c$ \\
\cline { 2 - 4 }$s_{i}=0$ (not contribute) & 0 & 0 & $v$ \\
\cline { 2 - 4 } & & &
\end{tabular}

\footnotetext{
4 This can be thought of as transferable utility, where the contributions equal $c \sum_{j=1}^{n} s_{j}$ with provision probability is $F\left(c \sum_{j=1}^{n} s_{j}\right)$, and where the units for $t$ are chosen such that we can ignore the $c$ in front of the summation.
} 
Let $s_{-i}=\left(s_{1}, \ldots, s_{i-1}, s_{i+1}, \ldots, s_{n}\right)$. Further denote $\operatorname{Pr}\left[\operatorname{piv} \mid s_{-i}, F\right]$ the probability that $i$ is pivotal given $s_{-i}$ and $F, \operatorname{Pr}\left[\operatorname{lost} \mid s_{-i}, F\right]$ the probability of a lost cause, and $\operatorname{Pr}\left[\mathrm{red} \mid s_{-i}, F\right]$ the probability of being redundant:

$$
\begin{aligned}
& \operatorname{Pr}\left[\operatorname{piv} \mid s_{-i}, F\right]=\sum_{x=1}^{\infty}\left(\operatorname{Pr}\left[C_{-i}=x-1 \mid s_{-i}\right] f(x)\right) \\
& \operatorname{Pr}\left[\operatorname{lost} \mid s_{-i}, F\right]=\sum_{x=1}^{\infty}\left(\operatorname{Pr}\left[C_{-i}<x-1 \mid s_{-i}\right] f(x)\right) \\
& \operatorname{Pr}\left[\operatorname{red} \mid s_{-i}, F\right]=\sum_{x=1}^{\infty}\left(\operatorname{Pr}\left[C_{-i}>x-1 \mid s_{-i}\right] f(x)\right) .
\end{aligned}
$$

These probabilities correspond to the likelihoods of the realized contributions equaling the respective column in the payoff matrix. It is a lost cause from individual $i$ 's point of view if, conditional on others' contributions, the public good will not be provided even if $i$ contributes. Her contribution is redundant if the public good is provided even if she does not contribute. Her contribution is pivotal if the public good is provided if she contributes but not provided if she does not contribute.

Given $s_{-i}$ and $F$, a player is willing to contribute if her expected payoff contributing exceeds that of not contributing:

$$
\begin{aligned}
& \operatorname{Pr}\left[\operatorname{lost} \mid s_{-i}, F\right](-c)+\operatorname{Pr}\left[\operatorname{piv} \mid s_{-i}, F\right](v-c)+\operatorname{Pr}\left[\operatorname{red} \mid s_{-i}, F\right](v-c) \\
& \quad \geq \operatorname{Pr}\left[\operatorname{red} \mid s_{-i}, F\right] v \Rightarrow \operatorname{Pr}\left[\operatorname{piv} \mid s_{-i}, F\right] \geq \frac{c}{v} .
\end{aligned}
$$

It follows that the decision rule for each $i$ is:

$$
s_{i}= \begin{cases}0 & \text { if } \operatorname{Pr}\left[\operatorname{piv} \mid s_{-i}, F\right]<\frac{c}{v} \\ 0 \text { or } 1 & \text { if } \operatorname{Pr}\left[\operatorname{piv} \mid s_{-i}, F\right]=\frac{c}{v} \\ 1 & \text { if } \operatorname{Pr}\left[\operatorname{piv} \mid s_{-i}, F\right]>\frac{c}{v}\end{cases}
$$

In words: $i$ should contribute if she perceives her chance of being pivotal is greater than the cost-to-value ratio.

Because the number of contributions in equilibrium is of greater interest than which players contribute in equilibrium, I will treat two equilibria with the same number of contributions as one equilibrium. Denote $C^{*}$ to be the number of contributors in equilibrium $s^{*}$. Notice that in equilibrium $s^{*}$, a contributing player believes with probability one that exactly $C^{*}-1$ others are contributing, so that the contributing player is pivotal with probability $f\left(\sum_{j \neq i} s_{j}^{*}+1\right)$, which equals $f\left(C^{*}\right)$. A non-contributing player is pivotal with probability $f\left(C^{*}+1\right)$.

Assuming that a player in a pure equilibrium who is indifferent between contributing and not contributing will contribute, the conditions for existence of an equilibrium $s^{*}$ are: 

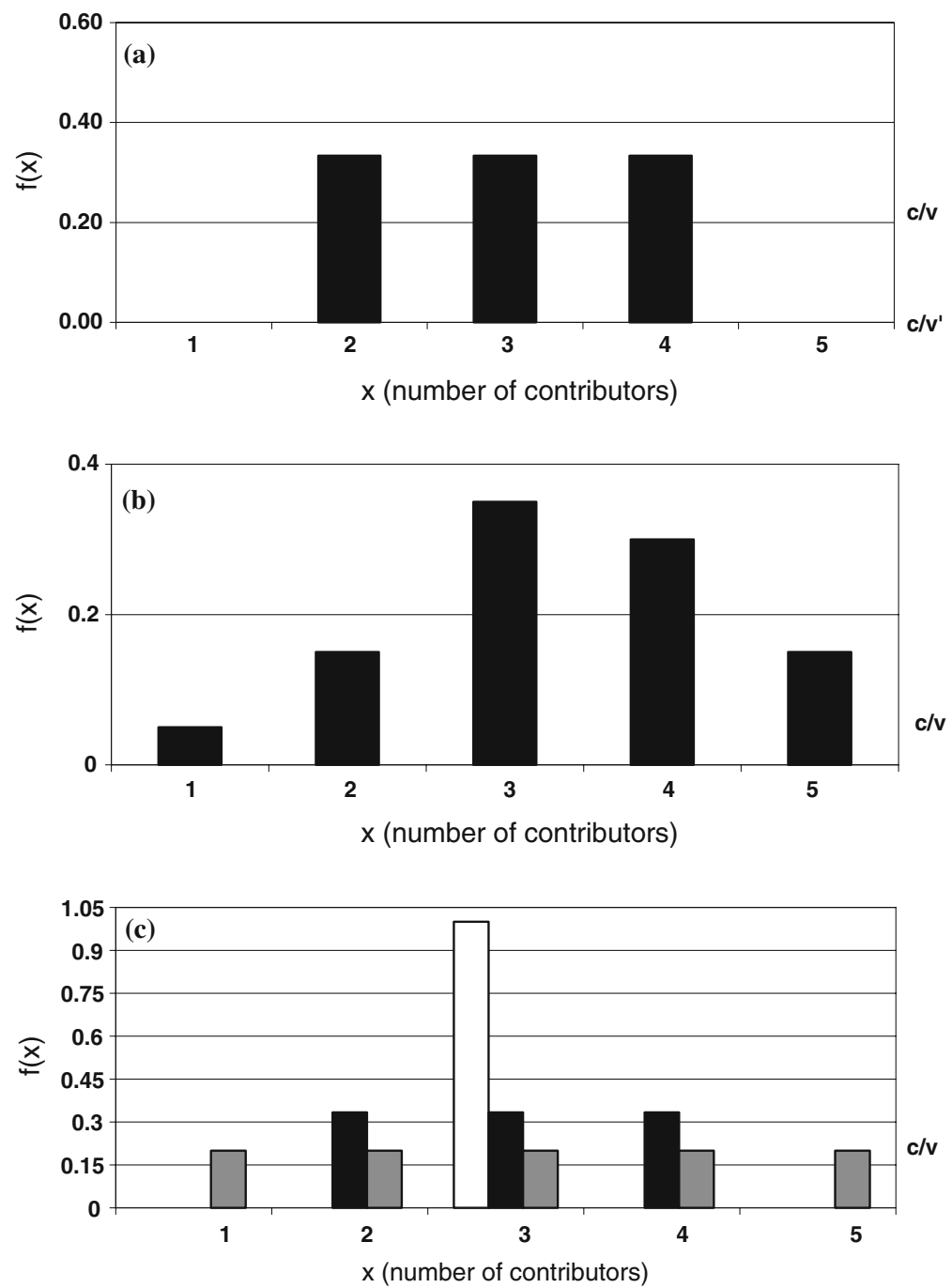

Fig. 1 Finding equiliabria graphically. a A uniform pdf, $\mathbf{b}$ a strictly unimodal pdf; $\mathbf{c}$ three uniform pdfs

$$
C^{*}= \begin{cases}0 & \text { if } f(1)<\frac{c}{v} \\ x \in\{1, . ., n-1\} & \text { if } f(x) \geq \frac{c}{v} \text { and } f(x+1)<\frac{c}{v} \\ n & \text { if } f(n) \geq \frac{c}{v}\end{cases}
$$

Figure 1a illustrates these conditions with the uniform threshold distribution

$$
f(x)= \begin{cases}\frac{1}{3}, & x=2,3,4 \\ 0 & \text { otherwise }\end{cases}
$$


Suppose $n=5$. With $\frac{c}{v}=0.4$, as illustrated by the horizontal line at 0.4 , we have $f(x)<\frac{c}{v}$ for all $x$. Thus, the only equilibrium has 0 contributions. However, with $\frac{c}{v^{\prime}}=0.2$, as illustrated by the lower horizontal line, we have $f(0)<0.2$ and $f(4)>0.2>f(5)$. In this case, there are two equilibria: a trivial one with 0 contributions and a non-trivial one with 4 contributions.

\subsection{Changes in uncertainty}

A key feature to notice is that an equilibrium with an interior contribution level (strictly between 0 and $n$ ) is located where the pdf is downward sloping and crosses the $\frac{c}{v}$-line. In Fig. 1a with $\frac{c}{v}<\frac{1}{3}$, this occurs at $C^{*}=4$. A second key feature is that if the pdf is uniform as in Fig. 1a or unimodal (single peaked) as in Fig. 1b, then there is only one such crossing.

These two features allow us to examine what happens as uncertainty changes. Consider Fig. 1c, which depicts the three different uniform pdfs that I use in my experiment. The solid black pdf is $f$ from Fig. 1a. The white pdf $f^{\prime}$ is

$$
f^{\prime}(x)= \begin{cases}1, & x=3 \\ 0 & \text { otherwise }\end{cases}
$$

This represents the case of the least amount of uncertainty (i.e., perfect certainty of $t$ ). The gray pdf $f^{\prime \prime}$ is

$$
f^{\prime \prime}(x)= \begin{cases}\frac{1}{5}, & x=1,2,3,4,5 \\ 0 & \text { otherwise }\end{cases}
$$

Among these three pdfs, $f^{\prime \prime}$ has the most uncertainty.

If $\frac{c}{v}=0.45$, then the unique non-trivial equilibrium under $f^{\prime}$ is $C^{*}=3$. If uncertainty increases to $f$, then the unique equilibrium has no contributions. In this case, an increase in uncertainty eliminates all contributions. If instead $\frac{c}{v}=0.3$, due to a higher valuation of the public good, then the unique non-trivial equilibrium under $f^{\prime}$ is still $C^{*}=3$, but now there is a unique non-trivial equilibrium $C^{*}=4$ under $f$. In this case, equilibrium contributions are actually higher under the wider uncertainty.

Why does this happen? Remember that the marginal value of a contribution depends on both the public good value and on the probability of being pivotal. If the product of these two factors is sufficiently high, then contributing is optimal. An increase in threshold uncertainty changes the probability of being pivotal: it decreases it for some contribution profiles but increases it for others. But what matters for the contribution decision is whether or not the product of pivotalness probability and public good value is sufficiently high, and a property of the equilibrium is that an increase in threshold uncertainty always (weakly) increases the probability of being pivotal when the public good is sufficiently high. ${ }^{5}$

\footnotetext{
5 Graphically, as uncertainty increases, the peak of the pdf drops but the tails of the pdf rise. If the $\frac{c}{v}$-line is sufficiently low, then it will cross the wider-uncertainty pdf in the fatter tail, and equilibrium contributions
} 
Figure 1c provides another example of this logic. As uncertainty changes from $f^{\prime}$ to $f$ with $\frac{c}{v}=0.3$, then contributions increase. But now if uncertainty further increases from $f$ to $f^{\prime \prime}$, still keeping $\frac{c}{v}=0.3$, then the equilibrium contributions decrease to 0 because the $\frac{c}{v}$-line is now entirely above the pdf. If instead $\frac{c}{v}=0.15$, then each increase in uncertainty from $f^{\prime}$ to $f$ to $f^{\prime \prime}$ results in an increase in equilibrium contributions from 3 to 4 to 5 .

I summarize by stating the primary prediction I will test in the laboratory experiment:

Prediction: If $v$ is sufficiently large ( $\frac{c}{v}$ sufficiently small), then an increase in threshold uncertainty will lead to an increase in contributions. But if $v$ is sufficiently small, then an increase in threshold uncertainty will lead to a decrease in contributions.

\section{Experimental design}

This experiment was conducted at the California Social Science Experimental Laboratory located on the campus of the University of California, Los Angeles (UCLA). All subjects are drawn from the UCLA student population. Each experimental session consisted of 4 practice rounds and 30 real rounds, ${ }^{6}$ and each session had either 25 or 30 students. All decisions were made over a computer network in a computer currency called "tokens." Subjects amassed tokens depending on the decisions and the factors determined by the computer. At the end of the session, subjects were paid US dollars according to a pre-announced token/dollar exchange rate.

In each round, the computer randomly and anonymously assigns the subjects into groups of five, and each subject is given one computer token. Each subject's computer then displays the public good value and the threshold distribution. Instead of using the term "threshold distribution," subjects are told that the threshold distribution is a range $T=\{\underline{t}, \ldots, \bar{t}\}$ from which the computer will randomly and uniformly select the true threshold. Subjects are told that the "threshold-met value" and "threshold range" are the same for all individuals and groups in the room.

Before deciding whether to keep (do not contribute) or spend (contribute) the one given token, each subject is asked to assign percentage probabilities to what the others in his or her group will do. Since each group has five subjects, each subject assigns probabilities to the following five events: exactly 0 others in the group spend, exactly 1 other spends, exactly 2 others spend, exactly 3 others spend, and exactly 4 others spend. Once the assigned percentages add up to 100 percent and the subject confirms the entry, the subject then makes the decision to keep or spend the one token. Tokens not spent in the current round cannot be spent in later rounds. Subjects are not allowed to communicate with any other subjects in the room during the practice or real rounds.

\section{Footnote 5 continued}

will thus be higher. Put differently, the equilibrium probability of being pivotal has increased and so contributions increase. On the other hand, if the $\frac{c}{v}$-line is too high so that it is above the peak of the wider-uncertainty pdf, then contributions will plummet.

6 The exception is the $8 / 21$ session which ended after 26 rounds. 
A subject's payment for a given round has two parts. The first payment is based on the accuracy of the reported beliefs, which is derived using a proper scoring rule. The exact formula is

$$
\frac{v}{2}\left(\left[b_{i t}\left(\operatorname{actual}_{t}\right)\right]^{2}-\frac{1}{2}\left(\left[b_{i t}(0)\right]^{2}+\left[b_{i t}(1)\right]^{2}+\cdots+\left[b_{i t}(4)\right]^{2}\right)\right)+\frac{v}{4}
$$

where $b_{i t}(e), e=0, \ldots, 4$, is the percent assigned by $i$ to the event that $e$ others spend, and $b_{i t}\left(\right.$ actual $\left._{t}\right)$ is the percent assigned to that $x$ that actually occurs. The highest this payment can be in a given round is $\frac{v}{2}$, and the lowest is 0 . The second payment in a given round is that resulting from the contribution decisions. Letting $C$ be the total realized contributions in the group, this payment for subject $i$ is:

$$
\begin{array}{cl}
v+1 & \text { if } C \geq t \text { and } s_{i}=0 \\
v & \text { if } C \geq t \text { and } s_{i}=1 \\
1 & \text { if } C<t \text { and } s_{i}=0 \\
0 & \text { if } C<t \text { and } s_{i}=1
\end{array}
$$

I consider five different value-threshold range $(v, T)$ combinations,

$$
\begin{aligned}
& (3,\{3\}),(3,\{1,2,3,4,5\}) \\
& (6,\{3\}),(6,\{2,3,4\}),(6,\{1,2,3,4,5\}),
\end{aligned}
$$

in a variety of different treatments. While $v$ was held fixed in each session, $T$ varied in some sessions. Whenever the range was changed, it was fixed for the first 15 rounds, then changed to another range, which was then held fixed for the rest of the session. Table 1a lists the expected equilibrium contribution levels under the different $(v, T)$-combinations assuming expected payoff maximization as in the model. It also lists the qualitative predictions: equilibrium contributions should be higher under $(3,\{3\})$ than $(3,\{1,2,3,4,5\})$, and they should be successively higher under $(6,\{3\}),(6,\{2,3,4\})$, and $(6,\{1,2,3,4,5\})$. Table $1 \mathrm{~b}$ lists the different treatments and the number of sessions per treatment. As stated earlier, these threshold ranges correspond to the distributions in Fig. 1c. Given the limited budget, a choice had to be made about which treatments to run. Because decreased contributions under wider uncertainty seems the more intuitive prediction, more sessions were run with $v=6$ as it is this treatment with the less intuitive prediction that contributions increase under wider uncertainty.

This design allows for testing the theoretical predictions. This basic set-up, including $n=5$ and uniform threshold range, matches that used in the previous experimental studies of threshold uncertainty mentioned earlier. This establishes continuity with the other studies. The uniform threshold range is the best way to model the threshold distribution since subjects understand a uniform distribution. The uniform threshold range also implies single-crossing for both pure and symmetric equilibria, and this single-crossing implies nice qualitative predictions of contribution movements with changes in uncertainty. The chosen parameters profiles will allow for high and low 
Table 1 Treatment and session descriptions

\begin{tabular}{|c|c|c|c|c|c|}
\hline \multicolumn{3}{|c|}{$\begin{array}{l}\text { (a) Proportion of contributions by parameter combinations } \\
\qquad v=3\end{array}$} & \multicolumn{3}{|l|}{$v=6$} \\
\hline & $T=\{3\}$ & $T=\{1,2,3,4,5\}$ & $T=\{3\}$ & $T=\{2,3,4\}$ & $T=\{1,2,3,4,5\}$ \\
\hline Pure equlibrium & 3 & 0 & 3 & 4 & 5 \\
\hline Qualitative prediction & Decrease & & Increase- & & \\
\hline
\end{tabular}

(b) Treatments and sessions

$\begin{array}{ll}\text { Treatment } & \text { Number of sessions } \\ (3,\{3\}) \text { to }(3,\{1,2,3,4,5\})^{\mathrm{a}} & 1 \\ (6,\{3\}) \text { to }(6,\{2,3,4\})^{\mathrm{a}} & 1 \\ (6,\{2,3,4\}) \text { to }(6,\{3\})^{\mathrm{a}} & 1 \\ (6,\{3\}) \text { to }(6,\{1,2,3,4,5\})^{\mathrm{a}} & 3 \\ (6,\{1,2,3,4,5\}) \text { to }(6,\{3\})^{\mathrm{a}} & 1 \\ (6,\{3\}) & 1 \\ (6,\{2,3,4\}) & 3 \\ (6,\{1,2,3,4,5\}) & 3\end{array}$

a When the threshold range switches, the first 15 , the rounds are under the first range, and the rounds are under the second range

$v$ and for high and low uncertainty. Data for all these scenarios are needed to compare with the predictions. Eliciting beliefs will allow for more direct testing of the underlying behavior of the subjects, and providing incentives to report true beliefs adds credibility to the beliefs data. ${ }^{7}$ Group sizes are held constant to remove the effects of group sizes on contribution levels. No information on others' decisions or payments is given and all decisions are made privately to remove social pressures or social comparisons that might affect behavior. ${ }^{8}$ The maximum payment for beliefs is half as much as the highest payment from the keep/spend decision. This should remove the motive for players to play a game that maximizes the beliefs payment.

Finally, I emphasize that the measure of pivotalness used in the analysis below refers to inferred pivotalness and not actual pivotalness. First, subjects cannot know their exact probability of being pivotal because they cannot know for sure ex ante exactly how many other contributions will be made. Second, subjects were not asked the likelihood of being pivotal. They were asked to report probabilities about possible outcomes, and then I infer a subjective probability of being pivotal from these elicited beliefs.

\footnotetext{
7 See Nyarko and Schotter (2002) and Hurley and Shogren (2005) for extended discussion on the benefits of using elicted beliefs data. I am not the first to use elicted beliefs data in public goods experiments (see Offerman 1996 and Offerman et al. 1996).

${ }^{8}$ Subjects are in a large room sitting at computer terminals. During the instructional phase of the experiment but before choices are made, the subjects are prompted to pull out dividers that make it impossible for one subject to observe another's computer screen without standing up and disrupting the experiment. This never happened, so privacy was maintained. Such dividers are commonly used in laboratory experiments to foster privacy.
} 
Table 2 Contribution means

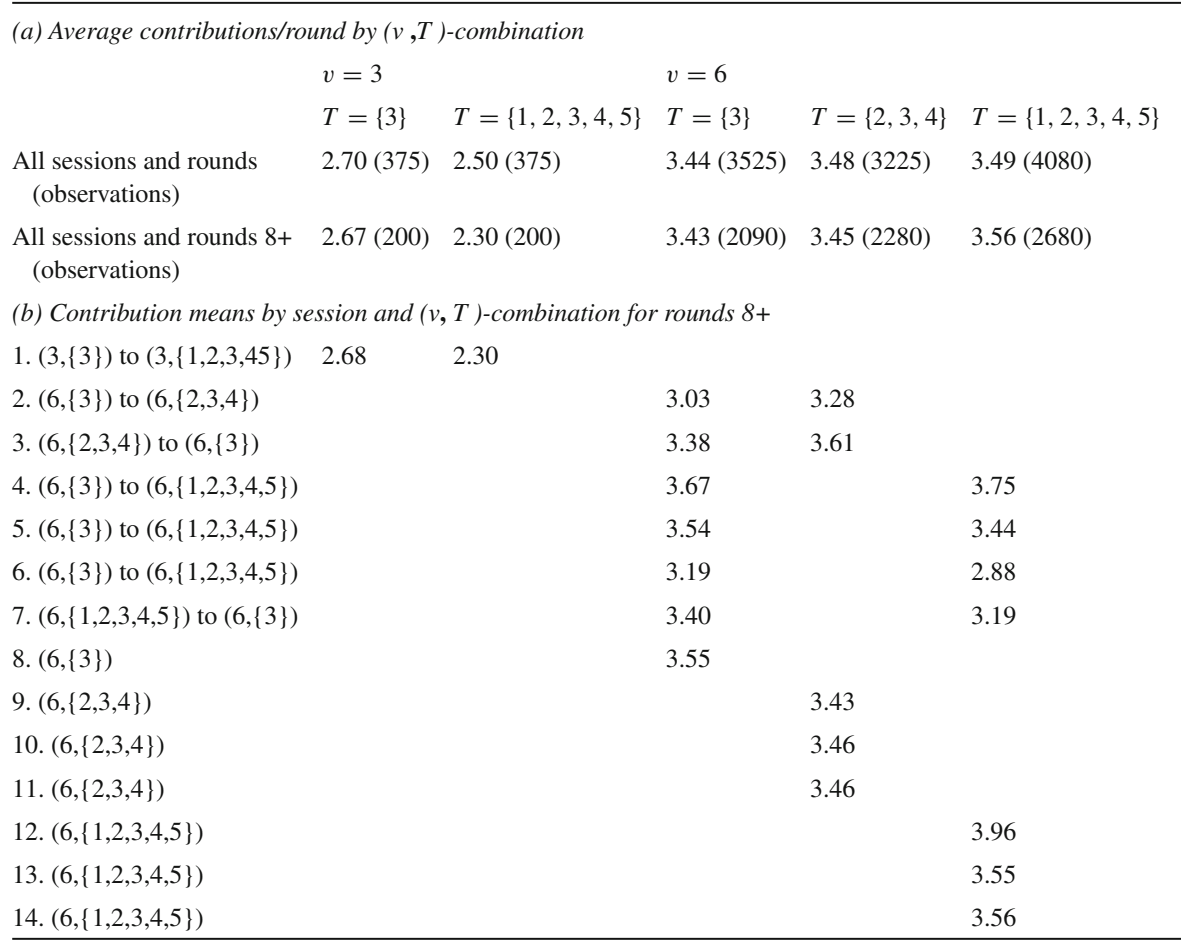

\section{Experimental results}

Findings 1-4 summarize the main results.

Finding 1 The prediction concerning contribution levels under different $(v, T)$-combinations is only moderately verified.

Table 2a lists the contribution levels by $(v, T)$-combination by all rounds and for rounds 8 and higher. The quantitative contribution levels differ substantially from the mixed equilibrium contribution levels in all case. However, when $v=3$, contributions are higher under $T=\{3\}$ than under $T=\{1,2,3,4,5\}$ as qualitatively predicted by the model. In the later rounds where the predictions may be more likely to be verified (e.g., due to convergence to an equilibrium), contributions are over 7 percent higher. This difference is only weakly significant; a test of equal means gives a test statistic ${ }^{9}$ of 1.50 . This weakly significant test statistic may be due to

9 The $t$-statistic for testing the equality of two means $p_{x}$ and $p_{y}$ is

$$
Z=\frac{\widehat{p}_{x}-\widehat{p}_{y}}{\sqrt{p_{0}\left(1-p_{0}\right)\left(\frac{n_{x}+n_{y}}{n_{x} n_{y}}\right)}},
$$

where $p_{0}=\frac{p_{x} n_{x}+p_{y} n_{y}}{n_{x}+n_{y}}($ Newbold 1995, 360). 
the small sample size for $v=3$. When $v=6$, contribution levels are higher under wider uncertainty as predicted, although the differences in levels vary depending on the comparison and are sometimes quite small. The difference between contributions under $\{3\}$ and $\{1,2,3,4,5\}$ (test statistic 1.92) and between $\{2,3,4\}$ and $\{1,2,3,4,5\}$ (test statistic 1.72) are moderately significant. Contributions under $\{3\}$ and $\{2,3,4\}$ do not statistically differ (test statistic 0.30 ). Unlike for the $v=3$ case, the sample sizes are quite large for these $v=6$ comparisons.

Table $2 \mathrm{~b}$ further breaks down contribution levels in the later rounds by session. Contribution means vary widely across sessions-even when under the same $(v, T)$ combination. Of the 7 sessions with multiple threshold ranges, contribution levels differ in the predicted manner in 4 and differ opposite of the predicted manner in $3 .{ }^{10}$ In the sessions where the range never changed, we again both match and do not match the predictions. As predicted, contributions under $\{1,2,3,4,5\}$ sessions are always higher than under $\{3\}$ and $\{2,3,4\}$, but contributions in the single $\{3\}$-session are slightly higher than under the three $\{2,3,4\}$-sessions.

In short, aggregated contribution levels under the various $(v, T)$-combinations differ qualitatively as expected in some cases but not all, and this verification is moderate at best. Figure 2 provides visual support for this conclusion. As predicted for the low value case, there does appear to be a clear downward trend in contributions in Session 1 (Fig. 2a) after uncertainty increases (signified by the vertical dotted line) at the start of round 16. However, upward or downward trends after the change in uncertainty are harder to spot visually within Sessions 2 through 7. The visual comparison across Sessions 8 through 14, which did not have changes in uncertainty, entails looking at levels across sessions. Again, it is difficult to tell visually that contributions are higher in one session than another. That said, the stronger result for Session 1 is likely due to the fact that the equilibrium prediction under the low public good value matches the naive guess that wider uncertainty hurts contributions. Under the high public good value, equilibrium pivotalness works against the naive intuition.

Because the verification of the prediction is moderate at best, two questions follow: why are the predictions verified to any degree, and why are they not verified to a larger degree? While there are many possible reasons, such as heterogeneity in subjects' innate cooperativeness (Burlando and Guala 2005), I explore these questions using the data on subjects' elicited beliefs. This allows me to focus directly on the strategic nature of the decision as it relates to pivotalness. Finding 2 provides additional justification for the use of my particular data.

\section{Finding 2 The reported beliefs move in manners consistent with beliefs-updating.}

Let $\overline{b_{i t}}=\sum_{e=0}^{n-1} e b_{i t}(e)$ be the mean of $i$ 's reported beliefs in period $t$. Although subjects are randomly matched in each round, it is likely that subjects use contribution levels of the prior rounds to help predict what current group members will contribute. In this case, $\overline{b_{i t}}$ will be closer to what happened in $t-1$ than $\overline{b_{i t-1}}$. It will also be true that the probability assigned in $t$ to the event that occurred in $t-1$ will be higher

\footnotetext{
10 In practice, the sessions within which the threshold range changes are the better ones to look at for testing my hypothesis because, as Camerer (1995) explains, the presence of individual, group, or session effects makes comparison across sessions more problematic.
} 

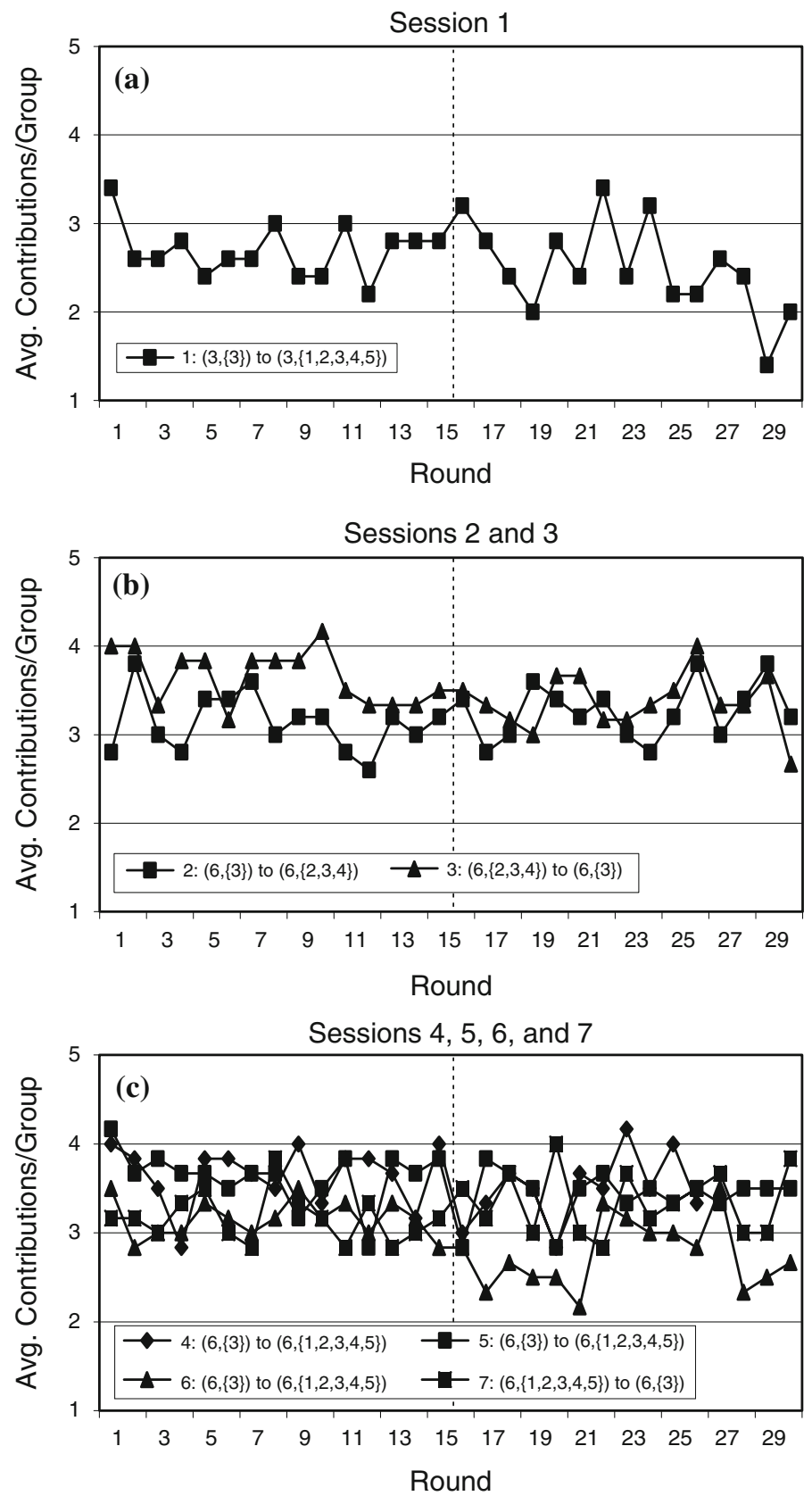

Fig. 2 Average contribution/group by round and session 

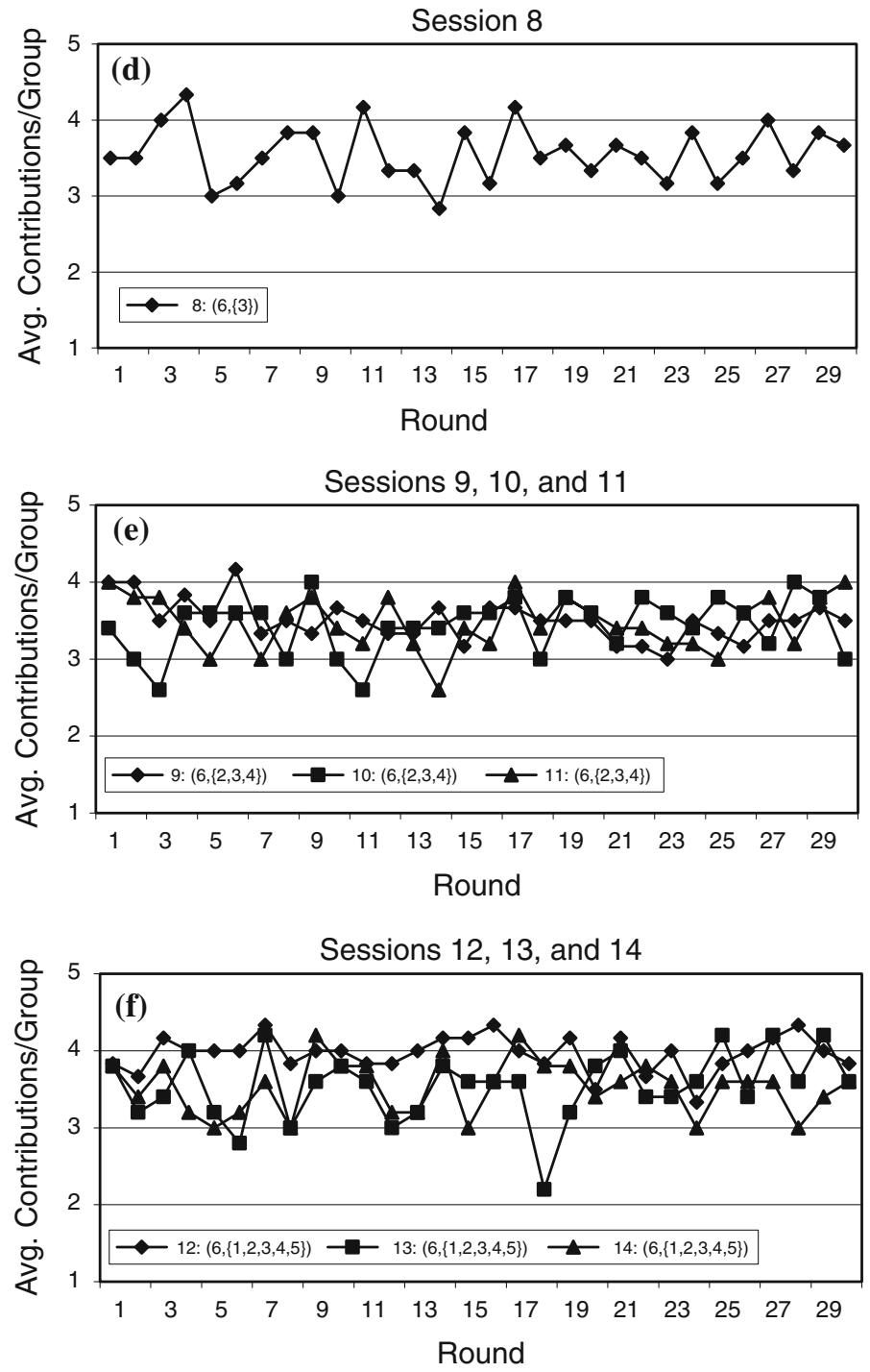

Fig. 2 continued

than the probability assigned to that event in $t-1$ (e.g., if $i$ assigned 30 percent to 3 others spending in $t-1$ and 3 others spent in $t-1$ then $i$ should assign 30 percent or more to 3 others spending in $t$ ).

Table 3 a lists how frequently reported beliefs moved in these two manners for each session. The first round of a particular parameter profile is not included in the calculation of this percentage. The averages moved as expected between 72 percent and 79 percent of the time across the sessions, and 75 percent overall. Subjects (weakly) 
Table 3 Measures of reported beliefs movements

\begin{tabular}{|c|c|c|c|}
\hline & $\begin{array}{l}\% \text { of time reported } \\
\text { beliefs average moved } \\
\text { towards last actual }\end{array}$ & & $\begin{array}{l}\% \text { of time assigned } \\
\text { higher percent to last } \\
\text { actual }\end{array}$ \\
\hline overall rounds $8+$ & $\begin{array}{l}0.75 \\
0.76\end{array}$ & & $\begin{array}{l}0.82 \\
0.82\end{array}$ \\
\hline 1. $(3,\{3\})$ to $(3,\{1,2,3,45\})$ & \multicolumn{2}{|l|}{0.76} & 0.79 \\
\hline 2. $(6,\{3\})$ to $(6,\{2,3,4\})$ & \multicolumn{2}{|l|}{0.76} & 0.85 \\
\hline 3. $(6,\{2,3,4\})$ to $(6,\{3\})$ & \multicolumn{2}{|l|}{0.79} & 0.85 \\
\hline 4. $(6,\{3\})$ to $(6,\{1,2,3,4,5\})$ & \multicolumn{2}{|l|}{0.78} & 0.83 \\
\hline 5. & \multicolumn{2}{|l|}{0.73} & 0.81 \\
\hline 6. & \multicolumn{2}{|l|}{0.78} & 0.80 \\
\hline 7. $(6,\{1,2,3,4,5\})$ to $(6,\{3\})$ & \multicolumn{2}{|l|}{0.79} & 0.85 \\
\hline 8. $(6,\{3\})$ & \multicolumn{2}{|l|}{0.77} & 0.82 \\
\hline 9. $(6,\{2,3,4\})$ & \multicolumn{2}{|l|}{0.75} & 0.82 \\
\hline 10. & \multicolumn{2}{|l|}{0.75} & 0.82 \\
\hline 11. & \multicolumn{2}{|l|}{0.74} & 0.81 \\
\hline 12. $(6,\{1,2,3,4,5\})$ & \multicolumn{2}{|l|}{0.75} & 0.82 \\
\hline 13. & \multicolumn{2}{|l|}{0.73} & 0.78 \\
\hline 14. & \multicolumn{2}{|l|}{0.77} & 0.85 \\
\hline \multicolumn{4}{|c|}{ (b) Regressions of mean beliefs on various controls (standard errors in parentheses) } \\
\hline & OLS & 1st AR(1) & 2nd AR(1) \\
\hline Intercept & $0.45(0.018)$ & $0.26(0.016)$ & $0.26(0.028)$ \\
\hline Mean belief $t-1$ & $0.83(0.006)$ & $0.90(0.006)$ & $0.89(0.010)$ \\
\hline Actual $_{t-1}-$ mean belief $t-1$ & $0.13(0.004)$ & $0.12(0.003)$ & $0.19(0.006)$ \\
\hline Parameter round & - & - & $0.00(0.002)$ \\
\hline $\begin{array}{l}\text { (Mean belief } t-1) \\
\quad \text { (parameter round) }\end{array}$ & - & - & $0.00(0.001)$ \\
\hline $\begin{array}{l}\left(\text { Actual }_{t-1}-\text { mean belief } t-1\right) \\
\quad(\text { parameter round })\end{array}$ & - & - & $-0.01(0.000)$ \\
\hline$R^{2}$ & 0.61 & 0.63 & 0.63 \\
\hline Durbin-Watson & 2.28 & - & - \\
\hline
\end{tabular}

increased the probability placed on the last period's event between 78 percent and 85 percent of the time, and about 82 percent overall. ${ }^{11}$

Table $3 \mathrm{~b}$ presents estimates from regressions of $\overline{b_{i t}}$ on different control variables. OLS regressions suffer from two potential problems. First, $\overline{b_{i t}}$ is bounded between 0 and 4. Examination of the data reveals that of the 10,990 lagged observations only 132 observations had $\overline{b_{i t}}=4,6$ had $\overline{b_{i t}}=0$, and one had incorrectly imputed values for $\overline{b_{i t}}$. Removing these 139 observations (less than 1.5 percent of the data) leaves

11 Offerman et al. (2001) report similar findings regarding elicted beliefs in their study. 
10,851 observations, and regressions on these data should not suffer from inconsistencies that could result from the censoring. The first estimates listed are from an OLS regression of $\overline{b_{i t}}$ on a constant, $\overline{b_{i t-1}}$, and $\left(\right.$ actual $\left._{t-1}-\overline{b_{i t-1}}\right)$ using this reduced set of observations. All estimates have the expected signs and are highly significant.

A second problem is that OLS does not account for possible autocorrelation, and the standard Durbin-Watson test indicates the presence of negative autocorrelation, as evidenced by a test statistic significantly different than 2 . Autocorrelation is detected even though this statistic should be biased towards 2 because of the lagged dependent variable. $^{12}$ Table $3 \mathrm{~b}$ presents results from two different 1 st-degree autoregressions. The first AR(1) gives results similar to the OLS results. The second AR(1) includes more control variables that capture how beliefs-updating might differ in later rounds. The $R^{2}$ values over 60 percent indicate that a significant amount of the mean beliefs can be explained by the regressors used. We observe that adding additional controls to the parsimonious specification does not add much explanatory power.

Finding 2 suggests that reported beliefs reflect the subjects' true beliefs. I also note that the computer interface did not list the subject's beliefs reports from prior rounds (it only lists contribution decisions and payments) when subjects report their beliefs in the current round. Thus, it appears that subjects' reported beliefs do reflect internalized beliefs since they are related from round to round without being listed on the computer screen.

With the use of these data now justified, I can combine the reported beliefs with the threshold distribution probabilities to directly calculate each $i$ 's subjective probability of being pivotal in time $t$ :

$$
\operatorname{Pr}\left[\operatorname{piv}_{t} \mid b_{i t}, T\right]=b_{i t}(0) \operatorname{Pr}[t=1 \mid T]+\cdots+b_{i t}(4) \operatorname{Pr}[t=5 \mid T] .
$$

Because the theoretical decision rule depends on a player's subjective beliefs about others' contributions, I can now use the reported beliefs to ascertain how closely the observed behavior reflects the game-theoretic decision rule that subjects contribute when $\operatorname{Pr}\left[\operatorname{piv}_{t} \mid b_{i t}, T\right] \geq \frac{c}{v}$ and do not contribute otherwise.

Finding 3 Letting reported beliefs proxy for true beliefs, subjects' actions are not consistent with expected payoff maximization.

Table 4 details how frequently subjects' contributions matched this decision rule. 65 percent of all decisions are consistent with expected payoff maximization. ${ }^{13}$ Only about 1 percent more are consistent in rounds 8 and higher. Note that deviations from the decision rule differ across sessions and $(v, T)$-combinations, with 55 percent to 80 percent consistent across sessions and 50 percent to 70 percent across $(v, T)$-combinations. When $v=6$ and the decision rule says " should not" contribute, then subjects are more likely to contribute, whereas when $v=3$ and the rule says " should not", then subjects are more likely to not contribute. These percentages are similar to the approximately two-thirds of subjects found to act consistently with expected payoff

\footnotetext{
12 See Chapter 13 in Greene (1997) for a discussion of autocorrelation and autocorrelation tests.

13 This calculation uses all observations except the one with the incorrectly reported beliefs, thus leaving a total of 9,629 observations.
} 
maximization by Offerman et al. (1996). Further observe that wider uncertainty alone does not drive the percentage consistent up or down. When $v=3$, fewer decisions are consistent with expected payoff maximization, while more are consistent when $v=6$. One plausible explanation is that an expected payoff calculation made by a subject in the wide uncertainty case with $v=3$ is much harder to make than under wide uncertainty with $v=6$. Yet, there is no way to confirm this conjecture using the data.

Finding 3 thus provides an initial answer to the second question posed above: the predictions are not strongly verified because subjects are not following the model's decision rule. The next finding suggests why the predictions are verified to any degree.

Finding 4 Subjects are more likely to contribute when they believe they are more likely to be pivotal.

Figure 3 a plots three non-parametric fits of the probability of contribution for different values of $\left(\operatorname{Pr}\left[\operatorname{piv} \mid b_{i t}, F\right]-\frac{c}{v}\right)$. I use the Epanechnikov kernel in the NadarayWatson kernel estimator under three different smoothing bandwidth parameters $h=0.025,0.1$, and 0.15 (Härdle 1990). ${ }^{14}$ Denoting $x=\operatorname{Pr}\left[\operatorname{piv} \mid b_{i t}, F\right]-\frac{c}{v}$, where $x$ ranges from -0.333 to 0.833 in the data, this estimator is

$$
m_{h}\left(X_{i}, h\right)=\frac{\frac{1}{(h)(\# \mathrm{obs})} \sum_{\mathrm{obs}} \frac{3}{4}\left(1-\left(\frac{x_{\mathrm{obs}}-X_{i}}{h}\right)^{2}\right) I\left(\frac{x-X_{i}}{h} \leq 1\right) a_{\mathrm{obs}}}{\frac{1}{(h)(\# \mathrm{obs})} \sum_{\mathrm{obs}} \frac{3}{4}\left(1-\left(\frac{x_{\mathrm{obs}}-X_{i}}{h}\right)^{2}\right) I\left(\frac{x-X_{i}}{h} \leq 1\right)}
$$

The curve labeled "EP Decision Rule" depicts the model's game theoretic decision rule. Figure $3 \mathrm{~b}$ plots the $h=0.1$ fit with 95 percent confidence intervals. ${ }^{15}$ The first thing to note is that subjects are more likely to contribute than not contribute even at many negative values of $\left(\operatorname{Pr}\left[\operatorname{piv} \mid b_{i t}, F\right]-\frac{c}{v}\right)$. This provides further evidence for rejecting the consistency of observed actions with the model's expected payoff maximization decision rule. Nonetheless, while expected payoff maximization is clearly rejected, note that Fig. 3a also reveals that the likelihood of contributing increases in $\left(\operatorname{Pr}\left[\operatorname{piv} \mid b_{i t}, F\right]-\frac{c}{v}\right)$. The slope of the non-parametric fit is positive, with the estimated probability of contributing increasing from below 0.5 at $\left(\operatorname{Pr}\left[\operatorname{piv} \mid b_{i t}, F\right]-\frac{c}{v}\right)=-0.4$ to about 0.75 at high values of $\left(\operatorname{Pr}\left[\operatorname{piv} \mid b_{i t}, F\right]-\frac{c}{v}\right)$.

\footnotetext{
14 A smaller bandwidth parameter implies that the only observations to receive weight are those closer to the point being estimated. While a smaller bandwidth implies greater precision in the sense of putting more weight on the more appropriate observations, if the bandwidth parameter is too small, then too few observations will given weight. By trial and error, I found $h=0.025$ to be the smallest bandwidth that still includes a meaningful number of observations for most point estimates.

15 To obtain better confidence intervals, I should compute bootstrap interval estimates. For statistical ease, however, I use the approximate confidence interval described by Härdle (1990), (100-101). The interval is $m_{h}(x) \pm\left(c_{\alpha} c_{K}^{1 / 2} \widehat{\sigma}(x)\right) / \sqrt{(n h \widehat{f}(x))}$, where $c_{\alpha}$ is the $100-\alpha$ quantile of the normal distribution, $c_{K}^{1 / 2}$ is a kernel constant, $\widehat{\sigma}(x)$ is the estimate of the standard deviation, and $\widehat{f}(x)$ is the estimate of the density. This confidence interval is hampered by a bias, but as we see from the graph, the bias must be large for consistency with EP maximization to be a legitimate possibility.
} 


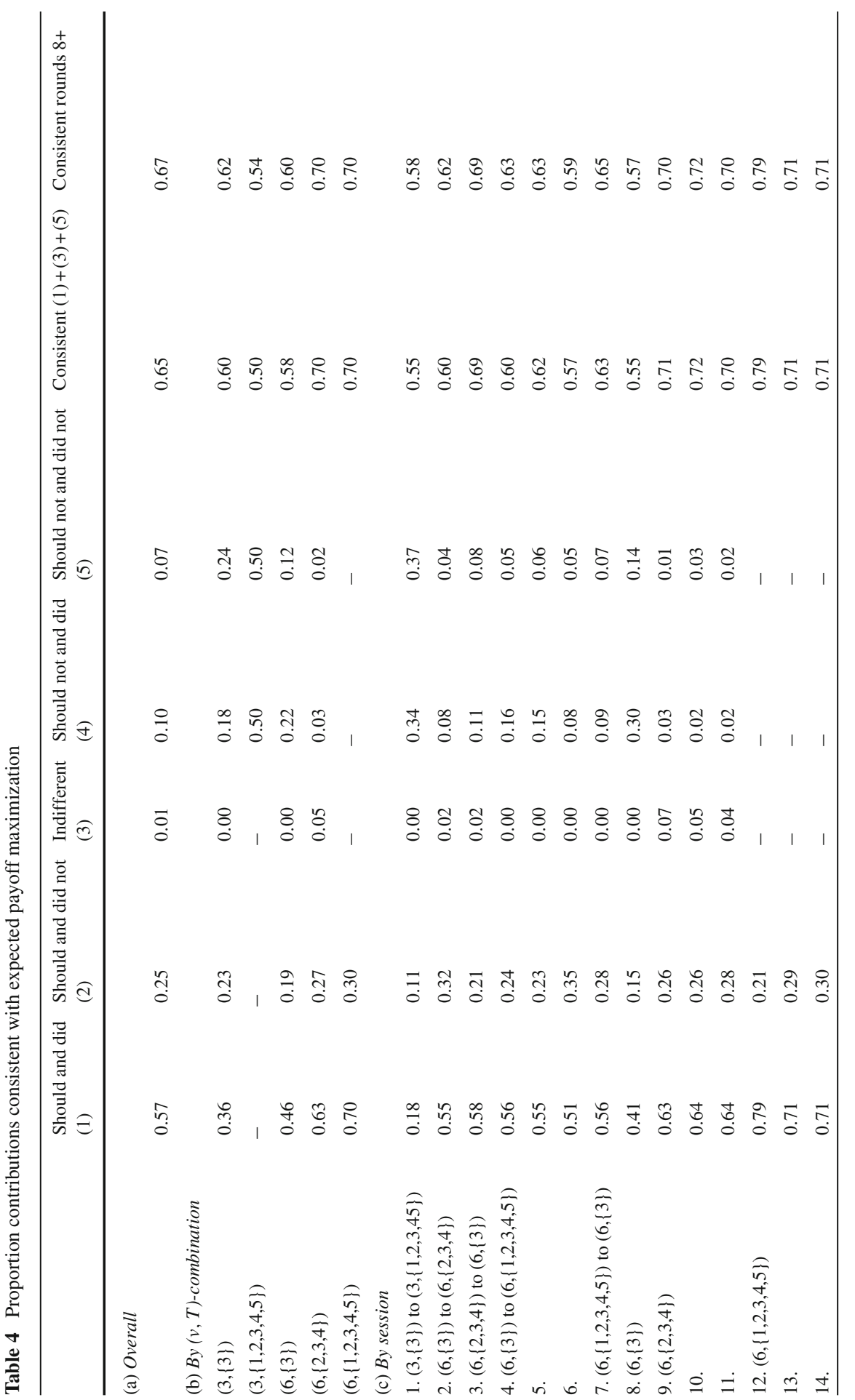



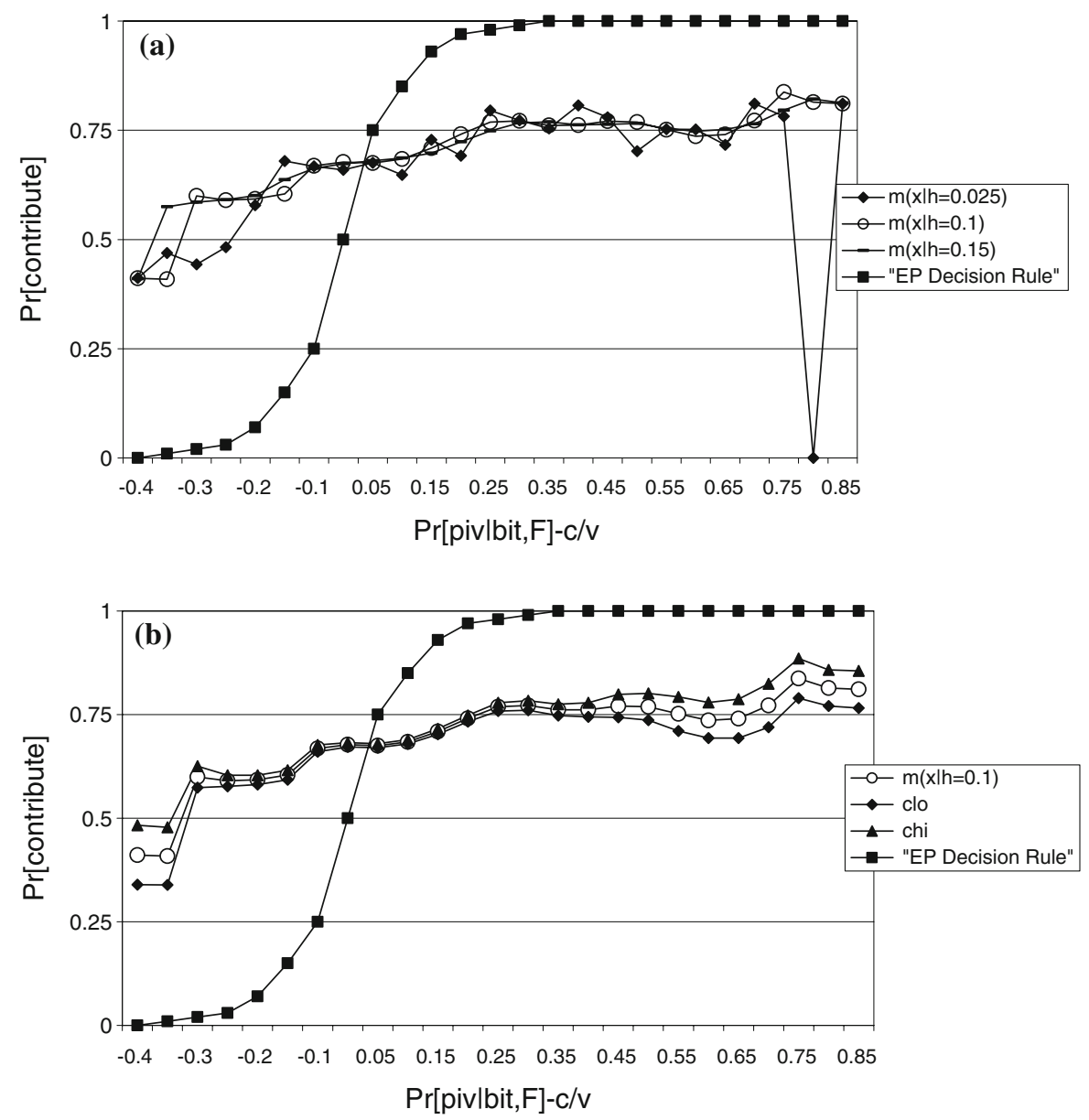

Fig. 3 Non-parametric regressions; a non-parametric EP regressions with $h=0.025,0.1,0.15$; b non-parametric EP regressions with $95 \%$ confidence interval for $h=0.1$

Overall, the subjects' actual decision rules and the model's decision rule have an important qualitative similarity and an important difference. The similarity is that subjects appear to strategically consider their pivotalness when making contribution decisions. Because pivotalness is strategic in the sense that it depends on a subject's beliefs about others' actions (in all cases except $T=\{1,2,3,4,5\}$ ), subjects are playing strategically in a game theoretic sense. Moreover, they are responding to pivotalness even in the presence of threshold uncertainty. Thus, the model captures an important aspect of the subjects' strategic behavior. This finding is particularly striking since subjects were not directly asked to report a probability of being pivotal. Had I asked them directly what the chances were that their own contribution was necessary to meet the threshold, then it is likely that this direct report of pivotalness would factor more heavily into their contribution decision, since directly asking them about pivotalness could unintentionally lead them to believe that pivotalness should 
determine the contribution decision. The fact that the inferred subjective pivotalness does matter suggests that subjects consider their pivotalness of their own volition.

However, the main difference between actual behavior and the model is that subjects do not respond as sharply to pivotalness around the cutpoint $\frac{c}{v}$ as implied by the model. When near the cutpoint, an increase in pivotalness only marginally increases the (global) probability of contribution. This offers one explanation for why contributions under $T=\{1,2,3,4,5\}$ were lower than under $T=\{3\}$ in sessions 5,6 , and 7. When $T=\{1,2,3,4,5\}$, a contributor's probability of being pivotal is $\frac{1}{5}$ no matter what she thinks others will do. When $T=\{3\}$, the probability of being pivotal is the probability assigned to the event that exactly two others contribute. If this probability is greater than $\frac{1}{5}$, which will often be the case, and if players use a best response rule that is strictly monotonically increasing in $\left(\operatorname{Pr}\left[\operatorname{piv} \mid b_{i t}, F\right]-\frac{c}{v}\right)$ (as in the non-parametrically estimated function in Fig. 3), then we will see more contributions under $T=\{3\}$ than $T=\{1,2,3,4,5\}$. More generally, if contributions depend not just on whether $\operatorname{Pr}\left[\operatorname{piv} \mid b_{i t}, F\right]$ is greater than $\frac{c}{v}$ but also on the difference between the two, then we will observe deviations from the model's predictions.

Why do subjects not follow the expected payoff maximization rule more closely? Though answering this question is not a purpose of this study, I can offer some possible answers. First, some subjects may not perfectly understand the decision making environment despite the efforts to teach them during the practice rounds. Second, subjects may be acting consistently but with preferences that are risk averse or risk loving. Third, subjects may have some form of other-regarding preferences. There could be still other explanations. As mentioned below, investigating these possibilities constitutes an important direction for future research.

\section{Discussion}

The theoretical results indicate that for highly valued public goods a widening of threshold uncertainty will increase individuals' probabilities of being pivotal, thereby driving up contributions. The experimental results provide only moderate support for these predictions. A widening of uncertainty often, but not always, results in movements in contributions in the expected manner. Although the subjects relate changes in threshold uncertainty into changes in pivotalness and consider pivotalness when making contribution decisions, they do not respond to pivotalness as sharply as the model implies. These last findings are obtained using data on subjects' subjective beliefs about other subjects' contributions.

The main implication of these findings is that whether or not threshold uncertainty hinders collective action will depend on the size of the benefits resulting from successful action and also on how individuals respond to pivotalness. Increases in threshold uncertainty may actually increase the likelihood of successful action when the benefits of successful collective action are large. However, because individuals do not respond to pivotalness to the degree implied by the model, this might only occur under small levels of threshold uncertainty. Threshold uncertainty will almost certainly hinder collective action when the value of the public good is low because wider uncertainty in this setting will lower individuals' probabilities of being pivotal. 
The risk of participating in a lost cause or of making a redundant contribution is then too high relative to the small potential gains.

It follows that groups facing threshold uncertainty will often need to undertake costly actions for collective action to succeed. One possibility would be the creation of mechanisms that exclude or punish non-participants. Another possibility, more in the spirit of this paper, would be the costly gathering of information that would reduce the variance in people's beliefs about the threshold, and this in turn raises a number of other strategic issues. For example, a group may actually prefer to not collect more information about the threshold if it is believed doing so will reduce the uncertainty so much that contributions will decrease. Also, a group leader with more precise information about the threshold may strategically reveal or not reveal her information in an attempt to obtain any surplus that can arise from contributions.

Future research should examine threshold uncertainty in these and other settings. Theoretical work should examine settings where individuals have private signals about the threshold, and an extension would allow some of those individuals to have noisier signals than others. Another setting would have a group leader who must choose whether or not to initiate costly information gathering. By examining these settings we can better understand how individuals' incentives to gather and share information differ across informational environments. Since much collective action occurs within formal groups or in the presence of other institutions, such work will lend insights into the actions taken by these groups to overcome the effects of threshold uncertainty.

A different direction of research should focus more closely on individuals' contribution decisions. That individuals do not respond as sharply to pivotalness suggests the presence of other strategic or behavioral factors in the decision making process. Prior research suggests a number of possibilities, e.g., that individuals differ in risk attitudes, dynamic strategic play, and learning. An alternative explanation is that subjects care about collective efficacy in addition to or instead of self efficacy (Kerr 1989; Lewinsohn-Zamir 1998). Examination of the experimental data reveals that subjects were less fearful of making redundant contributions (contributing to a near "sure thing") than contributing to a lost cause. This behavior could be evidence of collective efficacy concerns or risk aversion. Nonetheless, future work should account for these possibilities to better explain the observed behavior. ${ }^{16}$

Finally, more work should be done on reported beliefs. The very act of reporting beliefs can potentially lead a subject to act differently than if the beliefs were not reported. Measuring the extent of this possible bias would be useful as it would lend insights into possible biases in the beliefs data. These avenues of research will ultimately lead us to a more complete understanding of collective action.

\footnotetext{
16 This direction of research appears very promising. In preliminary work along these lines, I find evidence of statistically significant heterogeneity across individuals. While the simple expected payoff maximization rule is consistent with only 65 percent of decisions (Table 4), accounting for individual fixed effects in probit regressions yield esimates that correctly predict over 80 percent of decisions. Moreover, using a grid procedure to estimate risk aversion and cooperation bias parameters yields estimates that correctly predict about 90 percent of decisions. Another line of research would look at the presence of dynamic strategies.
} 
Open Access This article is distributed under the terms of the Creative Commons Attribution Noncommercial License which permits any noncommercial use, distribution, and reproduction in any medium, provided the original author(s) and source are credited.

\section{References}

Au W (2004) Criticality and environmental uncertainty in step-level public goods dilemmas. Group Dyn Theory Res Pract 8:40-61

Bagnoli M, Lipman B (1992) Private provision of public goods can be efficient. Public Choice 74:59-78

Bagnoli M, Lipman B (1989) Provision of public goods: fully implementing the core through private contributions. Rev Econ Stud 56:583-601

Budescu D, Rapoport A, Suleiman R (1995) Common pool resource dilemmas under uncertainty: qualitative tests of equilibrium solutions. Games Econ Behav 10:171-201

Burlando R, Guala F (2005) Heterogeneous agents in public goods experiments. Exp Econ 8:35-54

Camerer C (1995) Individual decision making. In: Kagel J, Roth A (eds) The handbook of experimental economics. Princeton University Press, Princeton, NJ, pp 587-703

Greene W (1997) Econometric analysis. Prentice-Hall Inc, Upper Saddle River

Gustafsson M, Biel A, Gärling T (1999) Overharvesting of resources of unknown size. Acta Psychol 103:47-64

Härdle W (1990) Applied nonparametric regression. Cambridge University Press, Cambridge, UK

Hurley T, Shogren J (2005) An experimental comparison of induced and elicted beliefs. J Risk Uncertain 30:169-188

Isaac RM et al (1989) The assurance problem in a laboratory market. Public Choice 62:217-236

Kerr N (1989) Illusions of efficacy: the effects of group size on perceived efficacy in social dilemmas. J Exp Soc Psychol 25:287-313

Ledyard J (1995) Public goods a survey of experimental research. In: Kagel J, Roth A (eds) The handbook of experimental economics. Princeton University Press, Princeton, NJ, pp 111-194

Lewinsohn-Zamir D (1998) Consumer preferences, citizen preferences, and the provision of public goods. Yale Law J 108:377-406

Marks M, Croson R (1998) Alternative rebate rules in the provision of a threshold public good: an experimental investigation. J Public Econ 67:195-220

McBride M (2006) Discrete public goods under threshold uncertainty. J Public Econ 90:1181-1199

Menezes F, Monteiro P, Temimi A (2001) Private provision of discrete public goods with incomplete information. J Math Econ 35:493-514

Newbold P (1995) Statistics for business and economics. Prentice-Hall Inc, Englewood Cliffs, NJ

Nitzan S, Romano R (1990) Private provision of a discrete public good with uncertain cost. J Public Econ 42:357-370

Nyarko Y, Schotter A (2002) An experimental study of belief learning using elicited beliefs. Econometrica 70:971-1005

Offerman T (1996) Beliefs and decision rules in public good games: theory and experiments. Tingergen Institute Research Series, Amsterdam, No. 124

Offerman T, Sonnemans J, Schram A (1996) Value orientations, expectations and voluntary contributions in public goods. Econ J 106:817-845

Offerman T, Sonnemans J, Schram A (2001) Expectation formation in step-level public good games. Econ Inq 39:250-269

Olson M (1965) The logic of collective action. Harvard University Press, Cambridge, MA

Palfrey T, Rosenthal H (1984) Participation and the provision of discrete public goods: a strategic analysis. J Public Econ 24:171-193

Palfrey T, Rosenthal H (1988) Private incentives in social dilemmas. J Public Econ 35:309-332

Palfrey T, Rosenthal H (1991) Testing game-theoretic models of free-riding: new evidence on probability bias and learning. In: Palfrey T (ed) Laboratory research in political economy. University of Michigan Press, Ann Arbor, MI, pp 239-268

Spencer M et al (2009) Rebate rules in threshold public good provision. J Public Econ 93:798-806

Suleiman R (1997) Provision of step-level public goods under uncertainty: a theoretical analysis. Ration Soc 9:163-187 
Suleiman R, Budescu D, Rapoport A (2001) Provision of step-level public goods with uncertain provision threshold and continuous contribution. Group Decis Negot 10:253-274

Wit A, Wilke H (1998) Public good provision under environmental and social uncertainty. Eur J Soc Psychol 28:249-256 